\title{
Concluding remarks: 15 years ago, now and in the near future
}

\author{
Andrea Milani \\ Department of Mathematics, University of Pisa, Piazza Pontecorvo 5, 56127 Pisa, Italy \\ email: milani@dm.unipi.it
}

\section{Highlights and conclusions}

For conclusions, the traditional approach in IAU meetings is to have a highlights speech, in which the most important points of the meeting are stressed. After consulting with the co-chairs of the Scientific Organizing Committee, I decided I would rather discuss the spirit of this meeting from a more conceptual point of view.

We think this meeting has been good, in that it has well outlined the state of the art on the subject of Near Earth Objects (NEOs), and this state of the art is in turn very good. However, to appreciate how good it is, we need to consider the evolution of the state of the art over some significant time span. In the opening remarks by Valsecchi we have been informed on the state of the art 236 years ago, when for the first time an object, comet D/1770 L1 Lexell, was discovered during a close approach to the Earth. We do not need to go that far. I would like to ask you to make the effort to go back, with your memory if you are as old as me, by reading the references if you are young, to 15 years ago, at the time of the Spaceguard Survey Report.

\section{Looking back}

At that time we had a lot of apparently good ideas, and most of them were wrong. Just a couple of examples: one is transport mechanisms. We have remembered in this meeting George Wetherhill: he was the one who stated the problem of trasport of meteorites and Near Earth Asteroids in the modern, scientific way, and in all his otherwise very successful scientific career he was never able to solve it, because he did not yet have the tools.

At that time, and I am speaking of 1991, not of the Middle Ages, we did not have any idea that a main belt asteroid, billions of years old, could nevertheless be on a chaotic orbit. We did not have any idea that the non gravitational perturbations, well known for artificial satellites, could play a significant role in the dynamical evolution of an asteroid. As for comets, we did not have the faintest idea that there was a source population, the Trans Neptunian Objects, of which we knew only one example, Pluto, which of course by itself is not a very good source of comets. We did not have an idea that asteroids could end up in the Sun, which we now know to be the main "sink" of NEOs.

Thus all our models on the origin of meteorites and NEOs were wrong. I do not want to offend anybody, of course many of you may claim to have written a precursor paper presenting some of the ideas later found to be right, but if we have to be honest, we have to count the times we have been right and also the ones in which we have been wrong, and the fact is, at that time we were more often wrong than right.

Other examples of things we did not know, at about that time: we did not have any example of binary asteroid, apart from some who turned out to be false (let us politely say "later not confirmed"). We did have radar ranging to asteroids, not radar images. 
We did not have very much in the way of spacecraft images either (Gaspra images were obtained in that same year 1991), thus we had to use Phobos images or, more often, Bill Hartmann's paintings to figure out what an asteroid would look like. Or maybe use, as Paolo Farinella et al. did, a Walt Disney comic strip to illustrate the concept of rubble pile.

On the subject of impact risk, we were aware of its existence, and we had already an idea of the order of magnitude of the probability of a catastrophic impact. Our population models, even for the largest NEA $(>1 \mathrm{~km})$, were only accurate within a factor 2, which was not bad for the tools we had at the time. The completeness levels were of the order of $10 \%$, and the surveys for discovering NEOs and other solar system objects were covering the sky, in a very incomplete way, up to a limiting magnitude 17.

Moreover, even after NEOs had been discovered, we did not have the mathematical tools to decide if they could impact the Earth in the medium terms, say 50 years. Still halfway through this period, in 1998, we could have a discussion among the best specialists of orbit determination which would not end with a consensus view on the possibility of an impact of a given object†.

\section{Progress achieved}

This was the level we were at the time: it was a young and immature science, which made rapid progress, as it is normal when you start from a low level of knowledge. If we look at the list of problems which have been solved in these 15 years we find it is very impressive. I have already mentioned the transport of NEAs and meteorites, the binaries and more in general the possible spin states of small bodies, the spacecraft and radar images and the consequent possibility to describe asteroids also in geomorphologic terms.

There has been an enormous increase of available data, especially after the beginning of operations of the automated CCD survey starting in 1998, but also because of spacecraft visits and of the availability of more powerful telescopes and instruments for physical observations. We can describe the increase by saying that we have about two orders of magnitude more objects known, and the number of objects for which we have other information besides the discovery has grown roughly in proportion.

We have also introduced the conceptual tools which have allowed to solve the problems. Our science has become mature: we can make predictions testing the theories and they turn out to be right, in most of the cases. There are a number of connections which were not even suspected and are now well understood. As an example, the connection between asteroid families, zodiacal light, meteorites and even traces of cosmic dust in geologic layers, was not even discussed much in this meeting because is now taken for granted. Another example is the transneptunians as source region for comets, including the Near Earth ones like Lexell: even after the discoveries of TNO started flowing in, at the beginning they were not considered a possible source.

An even more extreme example is meteor astronomy. Not so long ago it was regarded by most of our colleague astronomers as more witchcraft than science. The very idea that you could wait for many nights for something which may not even happen, and anyway could involve just grams of material, would be considered a waste of astronomical resources. Now even this is a mature science, which can successfully predict meteor showers and provide information on some comets and asteroids which is not available otherwise, short of a dedicated space mission; this is acheieved by using advanced technology, of which we

$\dagger 1997 \mathrm{XF}_{11}$. 
even had a glimpse in one exibition in the hall of this General Assembly $\ddagger$. Thus we have had plenty of new data because we worked hard, sometimes against a lot of difficulties, to obtain them, and we have used them overall very well.

There is also the issue of recognition within the astronomical community. We are having a significant recognition by the IAU. To have a NEO symposium at the IAU General assembly is not a small thing: there has never been an IAU meeting on such topic, and there has never been a Symposium on an even vaguely related topic at the previous General Assemblies.

\section{Complacency warning}

So, all is well in this brave new world for NEO science: no, this is not what I would like to say to conclude this Symposium. As people of my age know, being mature is a mixed blessing. By being mature, you tend to be more competent, but also to become complacent. To use a common, even abused, terminology, from the scientific revolution our science has undergone in the last 15 years we are passing to a stage of normal science. I have to tell you, even taking the risk of being unpleasant, that I have perceived, also in this meeting, symptoms of complacency, like a feeling of normal science. That is, very good science, using sound concepts, improving continously on what had been done before, but I do have the impression that the rate of discovery is slackening. Thus please look into yourself and ask yourself whether you are being complacent.

There are some symptoms of denial of annoying problems and discrepancies, generally small things which may or may not point to some weakness in our theoretical understanding and our models. There is the frequency of Tunguska-class events, with the additional outlier of the statistically too close approach of (99942) Apophis, which keeps coming back after we think we had solved the problem already. There are the size distribution of long period comets, and the physical aging of comets, still not properly constrained, with implications on the impact risk estimation which are small in relative sense, but may become large if the asteroid risk is taken care of. There is the issue of a suspected distribution of impacts on the Earth which may be statistically inconsistent with uncorrelated impacts. There are still ideas on the internal structure of asteroids which are at best to be considered theories, certainly not established facts. All minor disturbances with respect to the general success of the theories we have developed and carefully tested in recent years, still indications that we may have new and possibly important things to be discovered.

The reason for this is that we are in a transition phase. We have had a large increase in the availability of data, and we have had new conceptual tools, and this has fueled very rapid progress. However, unless our science receives a large inflow of fresh data, allowing us to find that our theories may be false, as well as true, we are at risk of decline.

\section{The foreseable future}

For our good luck, among the things which happened recently, there has been an important change of context for our specific discipline, and this is why we wanted to have the IAU Secretary General at the last session of this meeting. There has been widespread recognition, among the astronomical community but also among the political authorities in charge of funding science, of two important facts. One, NEOs are a subject

$\ddagger$ The exhibition showing the automated meteor observing station by the Ondřejov Observatory, Czech Republic. 
of science, and more specifically astronomical science. Two, the problem of NEO risk is responsability of the astronomers. Which means that the astronomers are entitled to ask for supplementary resources, on top of their normal reserach grants, to complete an activity which is useful to mankind, namely protecting our planetary environment from the imapct risk, but it is they who have to do it, to ask for the money and to use it to build the necessary astronomical resources. And if the astronomers are clever enough, they can build them in such a way that they can be efficiently used for multiple purposes, thus getting also a lot of science out of it.

This leads us to what is going to happen next, that is the next generation surveys, of which the first one, Pan-STARRS is expected to be operational in 2007. Thus the current transition period is going to be very short, it will be over soon: this is why this is the right moment for soul searching, to make sure we understand what are the things which are not clear, and which will be cast into doubt by the next very large inflow of data.

Three of these next generation surveys have been presented in this meeting $\dagger$ and apparently they have all acted very well to exploit this opportunity, being able to apply for sponsorship justified also by the mitigation of the NEO risk, but designing to perform multiple science with the same instrument and on the same images. In this way, NEOs are indeed a risk and an opportunity at the same time, as in the title of this meeting.

Thus I think there will soon be the opportunity to have a big boost in our science, and I would like to conclude by expressing my hope: that this period of normal science will just be a pause between two revolutions, and we will have the chance, together with younger people, to be involved a new phase of rapid progress as we have been actors in the previous one. 